\title{
Klotho negatively regulated aerobic glycolysis in colorectal cancer via ERK/ HIF1a axis
}

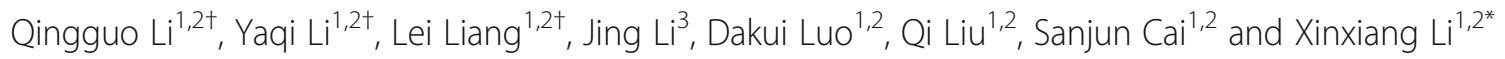

\begin{abstract}
Background: Klotho $(K L)$ was originally characterized as an aging suppressor gene, and has been identified as a tumor suppressor gene in a variety of cancers, including colorectal cancer. Recent years have witnessed the importance of metabolism transformation in cancer cell malignancies maintenance. Aberrant cancer cell metabolism is considered to be the hallmark of cancer. Our previous studies demonstrated that $\mathrm{KL}$ played negative roles in colon cancer cell proliferation and metastasis. However, its role in the cancer cell reprogramming has seldom been reported. The aim of this study was to examine the role of $\mathrm{KL}$ in aerobic glycolysis in colorectal cancer.
\end{abstract}

Methods: Combining maximum standardized uptake value (SUVmax), which was obtained preoperatively via a PET/CT scan, with immunohistochemistry staining, we analyzed the correlation between SUVmax and KL expression in colorectal cancer tissues. The impact of KL on glucose metabolism and its mechanisms were further validated in vitro and in vivo.

Results: Patients with lower $\mathrm{KL}$ expression exhibited higher ${ }^{18} \mathrm{~F}-\mathrm{FDG}$ uptake $(P<0.05)$, indicating that $\mathrm{KL}$ might participate in aerobic glycolysis regulation. In vitro assay by using colon cancer cell lines further supported this observation. By overexpressing KL in HTC116 and SW480 cells, we observed that the glycolysis was inhibited and the mitochondrial respiration increased, indicating that $\mathrm{KL}$ was a negative regulator of aerobic glycolysis. To seek for the underlying mechanisms, we tried to dig out the relation between KL and HIF1a signaling pathway, and found that KL negatively regulated HIF1a protein level and transcriptional activity. Western blot analysis showed that KL overexpression negatively regulated ERK pathway, and KL regulated aerobic glycolysis in part through its regulation of ERK HIF1a axis.

Conclusions: Taken together, KL is a negative regulator of aerobic glycolysis and KL inhibited glucose metabolism transformation via the ERK HIF1a axis.

Keywords: Colorectal cancer, Klotho, Aerobic glycolysis

\section{Background}

Colorectal cancer (CRC) is a common cancer and a leading cause of cancer-related mortality and morbidity worldwide [1]. The incidence of CRC is increasing annually and poses a great threat to the health care system. Despite significant progress in the diagnosis and treatment for CRC, the overall survival rate of patients varies from $90 \%$ to less than $5 \%$ due to different stages of the disease and inadequate

\footnotetext{
* Correspondence: 11491xx@sina.com

${ }^{+}$Qingguo Li, Yaqi Li and Lei Liang contributed equally to this work.

${ }^{1}$ Department of Colorectal Surgery, Fudan University Shanghai Cancer

Center, No.270 Dong'an Road, Xuhui District, Shanghai 200032, China

${ }^{2}$ Department of Oncology, Shanghai Medical College, Fudan University,

No.270 Dong'an Road, Xuhui District, Shanghai 200032, China

Full list of author information is available at the end of the article
}

diagnosis, and the mortality remains at a high level $[2,3]$. Therefore, there is an urgent need for new effective diagnosis and treatment targets and understanding the biology of CRC.

The klotho (KL) gene is a classical 'aging suppressor' gene. The role of KL was first demonstrated in the pathology of chronic kidney diseases $[4,5]$. The physiological and pathological function of KL shows that it can be used as a regulator of oxidative stress and senescence [6]. Recent studies in cancer demonstrated the KL could be inactivated by promoter hypermethylation and functions as a tumor suppressor [7-9]. KL was reported to be involved in the progression of a series of human cancers, including small cell lung cancer, breast cancer, 
hepatocellular carcinoma, ovarian cancer, and regulating tumorigenesis, proliferation, progression and resistance to traditional antitumor therapies [10-15]. The canonical roles of $\mathrm{KL}$ in cancer are to inhibit fibrosis and promote metastasis via the TGF, Wnt, IGF-I and the bFGF pathways [16-19]. In solid tumors, these signaling pathways are associated with stroma formation and hypoxic microenvironment shaping [20-24]. As is known, solid tumors possess a pronounced hypoxic tumor microenvironment, and these hypoxic conditions pose a threat to tumor cells due to reduced oxygen supply caused by limited vascular system. To survive under the severely hypoxic conditions, tumor cells must reprogram their metabolism pattern [25]. Otto Warburg discovered for the first time that solid tumor cells the metabolism within a solid tumor is significantly different from that of surrounding normal tissue. Over 50\% of the cellular energy is produced by glycolysis with the remainder being generated at the mitochondria in tumor cells. This shift occurs even when there is enough $\mathrm{O}_{2}$ present to support mitochondrial respiration. This phenomenon is termed as Warburg effect or aerobic glycolysis [26, 27]. The hypoxia-inducible factor 1 (HIF1) transcription factor is perhaps the most important aspect of how cells respond to hypoxic microenvironment and mediate aerobic glycolysis. The net result of hypoxic HIF1 activation is to shift energy production by increasing glycolysis and decreasing mitochondrial function [28]. HIF1 was initially discovered because of its response to low $\mathrm{O}_{2}$ concentrations, but now it becomes apparent that HIF1 can be regulated by other factors including oncogene activation or loss of tumor suppressor $[29,30]$. Aerobic glycolysis mediated by HIF1 not only provided proliferating cancer cells with building blocks for macromolecule synthesis and energy required in ATP form, but also created an acidic microenvironment caused by lactate that leads to destruction of extracellular matrix that favors metastasis. HIF1 regulated cancer cell proliferation, metastasis, and angiogenesis, thus it becomes a candidate therapeutic target in many cancers [31, 32].

Our previous study demonstrated that KL was a tumor suppressor in CRC and inhibited cell proliferation and metastasis, the malignancies of which were driven by and in part dependent on aerobic glycolysis [17]. However, no studies reported the connection between KL and aerobic glycolysis. Thus, we try to analyze the involvement of $\mathrm{KL}$ in aerobic glycolysis clinically and to uncover the underlying mechanisms of HIF1. These findings provide further insight for the anti-tumorigenic role of KL in CRC and raise the possibility that inducing $\mathrm{KL}$ expression and inhibiting aerobic glycolysis may provide novel treatment approaches for human colorectal cancer.

\section{Methods and materials}

\section{Patients and the whole body ${ }^{18} \mathrm{~F}$-FDG PET/CT protocol}

For PET/CT and Warburg study, 71 colorectal cancer patients who underwent radical surgery between January 2008 and December 2012 at Fudan University Shanghai Cancer Center (FUSCC) were included. Preoperative 18F-FDG PET/CT examination and histopathology confirmation of the presence of colorectal adenocarcinoma were conducted in all patients.

To investigate the mRNA level correlation between KL and HIF1 $\alpha$, a series of 61 patients who received radical resection of primary CRC were included in the study. The tumor samples were put in RNA later and stored in $-20{ }^{\circ} \mathrm{C}$ immediately after resection. The demographic and clinical characteristics of the above two cohorts are summarized in Additional file 1: Table S1.

The patients' in tissue microarray (TMA) has been described previously [33]. All patient studies in our research were approved by institutional review board of FUSCC.

\section{The whole body ${ }^{18} \mathrm{~F}$-FDG PET/CT protocol}

The whole-body FDG PET/CT was performed as previously described [24]. Briefly, ${ }^{18}$ F-FDG was made automatically by cyclotron (Siemens CTI RDS Eclipse ST; Knoxville, TN, USA) using an Explora FDG4 module. Patients had been fasting for more than $6 \mathrm{~h}$. Scanning was started $1 \mathrm{~h}$ after intravenous injection of the tracer $(7.4 \mathrm{MBq} / \mathrm{kg})$. The images were acquired on a Siemens biograph 16HR PET/CT scanner with a transaxial intrinsic spatial resolution of $4.1 \mathrm{~mm}$. CT scanning was first initiated from the proximal thighs to the head, with $120 \mathrm{kV}, 80-250 \mathrm{~mA}$, pitch 3.6, and rotation time $0.5 \mathrm{~s}$. Image interpretation was carried out on a multimodality computer platform (Syngo; Siemens). Quantification of metabolic activity was acquired using the standardized uptake value (SUV) normalized to bodyweight and the maximum SUV (SUVmax) for each lesion was calculated.

\section{Cell culture}

The human colon cancer cell lines HCT116 and SW480 were obtained from ATCC and cultured according to the standard ATCC protocols. In brief, HCT116 and SW480 cells were cultured in Dulbecco's Modified Eagle's Medium (DMEM), containing fetal bovine serum (FBS) in a final concentration of $10 \%$.

\section{RNA isolation and quantitative real-time PCR}

Total RNA was isolated by using TRIzol reagent (10,296,010, Invitrogen, USA), and TaKaRa's PrimeScript RT reagent (RR036A) was employed for reverse transcription to obtain cDNA samples. The expression status of designated genes and $\beta$-actin were determined by 
quantitative real-time $\mathrm{PCR}$ using an ABI $7900 \mathrm{HT}$ Real-Time PCR system (Applied Biosystems, USA). All reactions were run in triplicate. Primer sequences are listed in Additional file 1: Table S2.

\section{Protein extraction and western blot analysis}

Cells were washed twice with ice-dole PBS and lysed in RIPA buffer (150 mM NaCl, 1\% NP-40,,50 mM Tris/ $\mathrm{HCl}, \mathrm{pH} 8.0$ and $10 \%$ glycerol) supplemented with $100 \mu \mathrm{g} / \mathrm{ml}$ phenylmethylsulfonyl fluoride (PMSF) for $10 \mathrm{~min}$. Cell debris was removed by centrifugation at $12,000 \mathrm{rpm}$ for $20 \mathrm{~min}$ at $4{ }^{\circ} \mathrm{C}$. Protein concentrations were determined by using Thermo Pierce ${ }^{\circ}$ BCA Protein Assay Kit. $20 \mu \mathrm{g}$ total protein lysate was subjected to electrophoresis in denaturing 10\% SDS-polyacrylamide gel, and then transferred to a membrane for subsequent blotting with antibodies. KL antibody was obtained from Abcam (ab181373). Flag antibody was purchased from Sigma (F1804). Rabbit monoclonal antibody against ERK1/2 (9101), and phosphor-ERK1/2 (4370) were purchased from Cell Signaling Technology. $\beta$-actin (600081-lg), HIF1 $\alpha$ (20960-1-AP), HK2 (22029-1-AP), Glut1 (21829-1-AP), LDHA (19987-1-AP) antibodies that purchased from Proteintech.

\section{Lentivirus production and stable cell line selection}

The Flag-tagged coding sequences of human KL were cloned into pCDH-CMV-MCS-EF1-Puro plasmid (Systembio, SBI) to construct KL expression plasmid. In order to generate KL overexpression cell line, lentiviral particles were produced by co-transfection of pCDH-CMV-MCS-EF1-KL-Puro expressing constructs with psPAX2 and pMD2.G into HEK-293 T cells in a ratio of 4:3:1. Cell lines were obtained by infection of HCT116 and SW480 cells with lentiviral particles followed by puromycin selection.

\section{Immunohistochemical staining (IHC)}

Immunohistochemical staining of paraffin-embedded tissues with antibodies against KL and HIF1 $\alpha$ were performed to detect their expression according to standard procedures described previously [34]. In brief, paraffin-embedded sections were routinely baked overnight at $58{ }^{\circ} \mathrm{C}$, de-paraffinized in xylene, rehydrated through graded ethanol, quenched for endogenous peroxidase activity in $0.3 \%$ hydrogen peroxide and processed for antigen retrieval by high pressure cooking in citrate buffer $(\mathrm{pH}=6.0)$. Samples were incubated with primary antibodies overnight at $4{ }^{\circ} \mathrm{C}$ and secondary antibody for $1 \mathrm{~h}$ at room temperature. Diaminobenzidine (DAB) substrate was used for sample immunostaining. Subsequently, sections were counterstained with hematoxylin (Sigma). Anti-KL antibody (Abcam, ab181373) was used in a dilution of 1:100. HIF1 $\alpha$ antibody (Proteintech, 20,960-1-AP) was used in a dilution of 1:50. The immunohistochemically stained tissue sections were scored separately by two pathologists blinded to the clinicopathological parameters. The staining intensity was scored as 0 (negative), 1 (weak), 2 (medium) or 3 (strong). Extent of staining was scored as $0(<5 \%)$, 1(5-25\%), 2 (26-50\%), 3 (51-75\%) and 4 (>75\%) according to the percentages of the positive staining areas in relation to the whole carcinoma area. Scores for staining intensity and percentage positivity of cells were then multiplied to generate the immunoreactivity score (IRS) for each case. Samples having a final staining score of $\leq 4$ were considered to be low and those with score of $>4$ were considered to be high.

\section{Glycolysis analysis}

Glucose Uptake Colorimetric Assay Kits (Biovision) and Lactate Colorimetric Assay Kits (Biovision) were purchased to examine the glycolysis process in colon cancer cells, according to the manufacturer's protocols.

\section{Oxygen consumption rate (OCR) and extracellular acidification rate (ECAR)}

Cellular mitochondrial function and glycolytic capacity were measured using the Seahorse Bioscience XF96 Extracellular Flux Analyzer, according to the manufacturer's instructions of seahorse XF Cell Mito Stress Test Kit or Glycolysis Stress Test Kit. Cells were plated in XF96 Cell Culture Microplates (Seahorse Bioscience) at an initial cellular density of $4 \times 10^{4}$ cells/well the day before determination. Seahorse buffer consists of DMEM medium, phenol red, $25 \mathrm{mM}$ glucose, $2 \mathrm{mM}$ sodium pyruvate, and $2 \mathrm{mM}$ glutamine. For ECAR measurement, $10 \mathrm{mM}$ glucose, $1 \mu \mathrm{M}$ oligomycin, and $100 \mathrm{mM}$ 2-deoxy-glucose (2-DG) were automatically added to measure ECAR value. After monitoring baseline respiration, $1 \mu \mathrm{M}$ oligomycin, $1 \mu \mathrm{M}$ FCCP, and $1 \mu \mathrm{M}$ rotenone were automatically injected into XF96 Cell Culture Microplates to measure the OCR. The ECAR and OCR values were calculated after normalization of cell number. All experiments were performed in triplicate.

\section{Analysis of ATP production}

The ENLITEN ATP Assay System (Promega, FF2000) was used according to the manufacturer's instructions. Cells were harvested by scraping and were re-suspended in PBS. The cell suspension was divided into unequal aliquots. Part of the cell suspension was mixed with 5\% trichloroacetic acid (TCA). The remaining cells were used for the cell number calculation. Tris-acetate buffer ( $\mathrm{pH}$ 7.75) was then added to neutralize the TCA and to dilute the TCA to a final concentration of $0.1 \%$. The diluted sample $(40 \mathrm{~mL})$ was added to an equal volume of $\mathrm{rL} / \mathrm{L}$ reagen. Then, luminescence was measured. The ATP standard was serially diluted to generate a 
regression curve for calculating ATP concentrations in individual samples. The relative ATP concentration was determined and normalized to that of the control cells, which was designated as 1 . All experiments were performed in triplicate. Data is represented as mean \pm SD.

\section{Hypoxia response element (HRE) promoter activity with dual luciferase assay}

HEK-293 T Cells were seeded into 96-well culture plates and transfected by using Lipofectamine $\mathrm{e}^{\mathrm{mi}} 2000$ (Invitrogen). 200 ng of pCDH-CMV-MCS-EF1-KL-Puro expressing vector, HRE-luciferase plasmid (Addgene, 26,731) and the Renilla luciferase expression vector, pRL-TK (Promega), were transfected into cells [35]. Forty-eight hours after transfection, cells were assayed for both firefly and renilla luciferase activities using a dual-luciferase system (Promega), as described according to the manufacturer's protocol. All experiments were performed in triplicate. Data is represented as mean $\pm \mathrm{SD}$.

\section{Statistical analyses}

Statistical analyses were performed by SPSS software (version 17.0, IBM Corp., Armonk, NY, USA) using independent $t$ test (for continuous variables) and Pearson's $\mathrm{X}^{2}$ tests (for categorical variables). Statistical significance was based on two-sided $p$ values of $<0.05$.

\section{Results}

$\mathrm{KL}$ expression is negatively correlated with ${ }^{18} \mathrm{~F}-\mathrm{FDG} \mathrm{PET} /$ CT SUVmax value

${ }^{18}$ F-FDG PET/CT, which allows visualization of the metabolic activity of viable tumor cells, has been widely used in the management of cancer diagnosis. SUV $\mathrm{Smax}_{\max }$ has been widely used as a surrogate marker for the prognosis of numerous types of cancer, including colorectal cancer. In order to explore the clinical relationship between $\mathrm{KL}$ and glucose metabolism, we first examine the correlation between KL IHC staining and PET/CT $\mathrm{SUV}_{\text {max }}$ value. The representative pictures of $\mathrm{KL}$ staining were shown in Additional file 2: Figure S1. As expected, colorectal cancer patients with decreased expression of KL exhibited a higher $S_{U} V_{\text {max }}$ value (Fig. 1a). Through enlargement of the patients' sample, we confirmed that the correlation was of statistical significance (Fig. 1b). These results indicate that KL plays a certain negative role in glucose metabolism in colorectal cancer patients.

\section{$\mathrm{KL}$ inhibited aerobic glycolysis in colorectal cancer cells}

It is generally perceived that proliferated solid cancer cells shifted their glucose metabolism pattern to a hypoxic glycolysis manner. Based on the observation obtained from PET/CT, we suppose that KL may participate in the regulation of aerobic glycolysis. First, we overexpressed KL in HCT116 and SW480 cells, the effect was assayed and confirmed by quantitative PCR and immunoblot (Fig. 2a and b). Second, we examined glucose uptake, lactate production and ATP production, three primary indicators of the Warburg effect. As expected, KL decreased glucose uptake, lactate production and ATP production, indicating its inhibitory role in glycolysis (Fig. 2c-e).

Third, by using Seahorse XF Extracellular Flux Analyzers, we examined the impact of KL overexpression on glycolysis, as reflected by extracellular acidification rate or ECAR. ECAR is an indicator of acidification of the medium surrounding cancer cells that caused by lactic acid, which is a product of aerobic glycolysis. In KL overexpressed HCT116 and SW480 cells, the ECAR decreased significantly, reflecting the negative role of $\mathrm{KL}$ in extracellular acidification $(P<0.05)$ (Fig. 2f). Oxygen consumption by cells reflects mitochondrial respiration and could be measured by oxygen consumption rate,

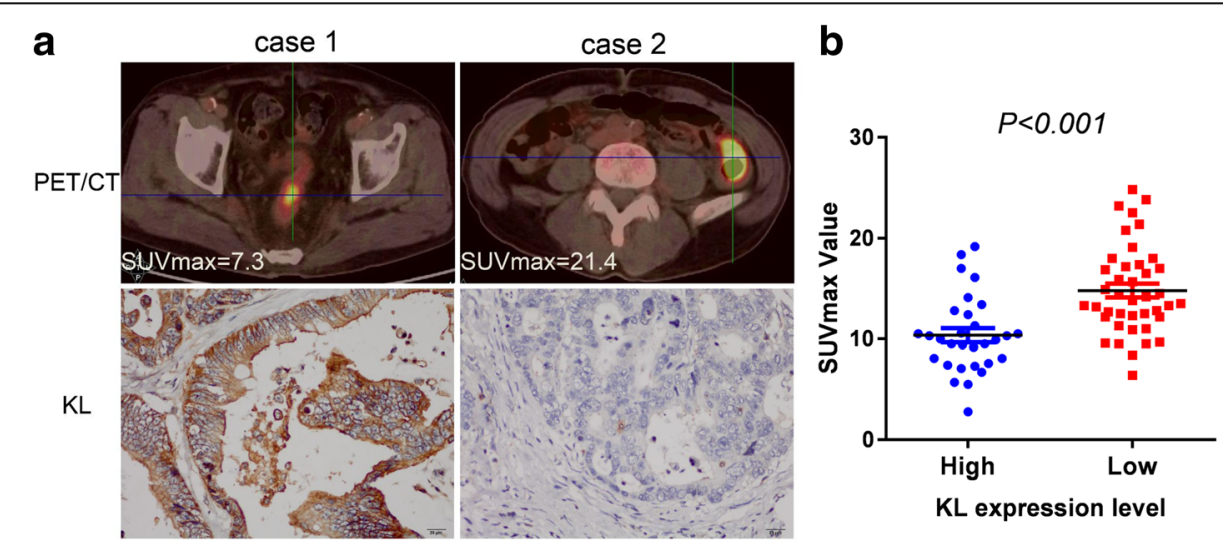

Fig. $1 \mathrm{KL}$ expression is negatively correlated with ${ }^{18} \mathrm{~F}$-FDG PET/CT SUVmax value. In order to assess the contribution of KL expression on metabolic burden, we evaluated the correlation between KL expression and SUVmax value obtained from PET/CT scanning by IHC staining. Representative images from PET/CT scanning in patients with low or high $\mathrm{KL}$ expression (magnification scale bar, $20 \mathrm{~mm}$ ) (a). Analysis of the correlation between SUVmax value with $\mathrm{KL}$ expression in KL-Low and KL-High groups of patients $(n=71, p<0.001)$ (b) 


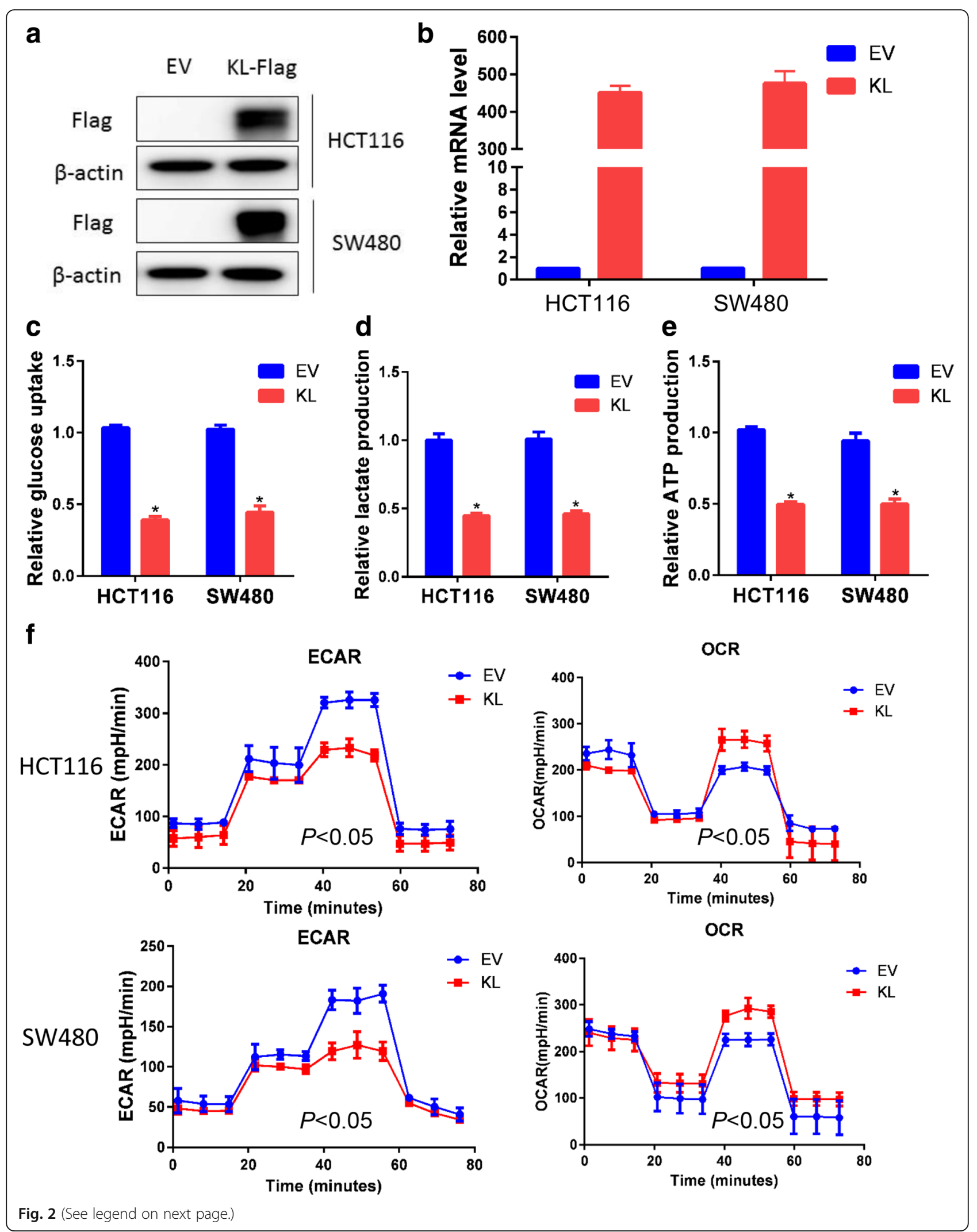


(See figure on previous page.)

Fig. $2 \mathrm{KL}$ inhibited aerobic glycolysis in colon cancer cells. With the aim to assess the role of KL in glycolysis regulation in vitro, as performed a series of in vitro assay. KL overexpressed CRC cancer cells were obtained by using lentiviral mediated transfection, and the efficacy was validated by using immunoblotting with FLAG antibody (a) and quantitative PCR (b). Forced expression of KL impairs glycolysis in colon cancer cells as determined by reducing glucose consumption (c), lactate production (d), and ATP production (e).Next, the impact of KL on glycolysis rate was assessed by using Seahorse Energy Flux system through examination of ECAR, which reflects the glycolytic rate, and the result suggested that KL overexpression inhibited glycolysis. Mitochondrial respiration, reflected by OCR, usually impaired by glycolysis, and OCR results showed that OCR value increased in $\mathrm{KL}$ overexpressed colon cancer cells, further indicating the negative role of $\mathrm{KL}$ in glycolysis ( $\mathrm{f}$. ${ }^{*} P<0.05$

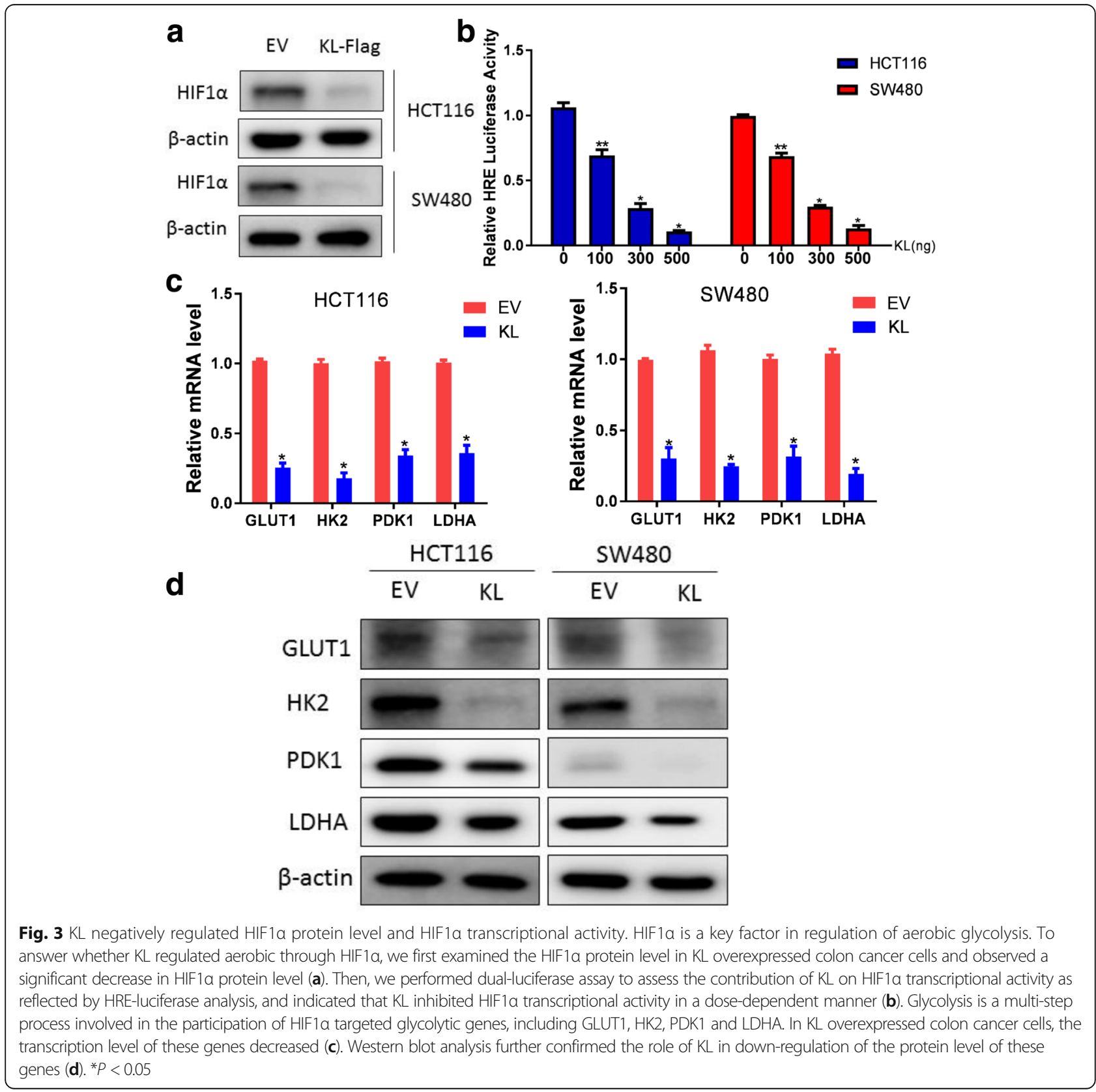


namely OCR. In the process of aerobic glycolysis, cells decrease the oxygen consumption rate. In consistence with the ECAR results, we observed a significant increase in the OCR value in KL overexpressed CRC cells $(\mathrm{P}<0.05)$, further reinforced the negative role of $\mathrm{KL}$ on aerobic glycolysis (Fig. 2f).

\section{KL negatively regulated HIF1a protein level and HIF1a transcriptional activity}

HIF1 $\alpha$ is a master regulator of aerobic glycolysis and hypoxia adaptation for solid tumors. To assess whether KL regulated aerobic glycolysis via its regulation of HIF1 $\alpha$, we first measured the protein level of HIF1 $\alpha$ in KL overexpressed CRC cells. As illustrated, HIF1 $\alpha$ protein level decreased significantly when $\mathrm{KL}$ expression was introduced (Fig. 3a). Next, we assessed the impact of KL on HIF1 $\alpha$ transcriptional activity as reflected by HRE-luciferase activity. We observed that KL negatively regulated HIF1 $\alpha$ transcriptional activity in a dose-dependent manner, reflecting a negative role of KL in HIF1 $\alpha$ pathway regulation (Fig. $3 b$ ). HIF1 $\alpha$ regulated aerobic glycolysis via transcription regulation of a series of glycolytic genes, including GLUT1, HK2 and LDHA. Thus, we examined the expression status of these glycolytic genes in KL overexpressed CRC cells. In consistence with the glycolysis analysis, GLUT1, HK2 and LDHA decreased in KL overexpressed CRC cells (Fig. 3c). Western blot analysis further validated the role of $\mathrm{KL}$ in regulation of these glycolytic genes (Fig. 3d).

\section{KL negatively correlated with HIF1a in CRC patients}

To support the in vitro observations, we measured the expression status of $\mathrm{KL}$ with HIF1 $\alpha$ in CRC patients. First, we measured the mRNA level correlation between KL and HIF1 $\alpha$ in a series of 61 patients, and observed no significant correlation (Fig. 4a). Then, we examined the correlation between KL and HIF1 $\alpha$ in protein levels by IHC staining in TMA including 185 patients. The detailed information of these patients has been described previously (Additional file 3) [33]. As exhibited, HIF1 $\alpha$ protein level was higher in patient with decreased KL expression (Fig. 4b). Moreover, the relevance of clinical significance, further supported the negative role of $K \mathrm{~L}$ in HIF1 $\alpha$ signaling pathway regulation (Fig. 4c).

\section{$\mathrm{KL}$ regulates HIF1a in an ERK dependent manner}

Based on the above observation that KL correlated with HIF1 $\alpha$ protein level in CRC patients instead of transcriptional level, we suppose that KL might regulate HIF1 $\alpha$ stability. First, we examined the half-life of HIF $1 \alpha$ in KL overexpressed CRC cells. As observed, HIF1 $\alpha$ half-life was significantly shorter than that in control parent cells, indicating a negative role of KL in HIF1 $\alpha$ stability (Fig. 5a and b). Mounting evidence has pointed out that ERK activation was responsible for HIF1 $\alpha$ stability maintenance. Then we examined the status of ERK signaling pathway in $\mathrm{KL}$ overexpressed CRC cells. We found that KL overexpression inhibited the activation of ERK (Fig. 5c). To determine whether KL regulated HIF1 $\alpha$, we overexpressed constitutive active form of ERK-MAPK kinase ERK2 $\left(\mathrm{ERK} 2^{\mathrm{E} 322 \mathrm{~K}}\right.$ ) into colon cancer cells. As exhibited, ERK2 $2^{\text {E322K }}$ introduction attenuated the decrease in HIF1 $\alpha$ protein level caused by $\mathrm{KL}$ overexpression, indicating that ERK pathway activation is responsible for the regulation of HIF1 $\alpha$ by KL (Fig. 5 d). Subsequently, we examined the effect of ERK2 ${ }^{\mathrm{E} 322 \mathrm{~K}}$ on glycolysis in KL overexpressed cells and found that $\mathrm{ERK} 2^{\mathrm{E} 322 \mathrm{~K}}$ transfection could eliminate the inhibit effect of KL on glycolysis (Fig. 5e). Moreover, we examined the expression status of glycolytic genes to assess the impact of ERK on KL/HIF1 $\alpha$ axis. As shown, $\mathrm{ERK} 22^{\mathrm{E} 322 \mathrm{~K}}$ introduction alleviated the attenuation on the expression of glycolysis genes caused by KL, further supporting our hypothesis that KL regulated HIF1 $\alpha$ via ERK
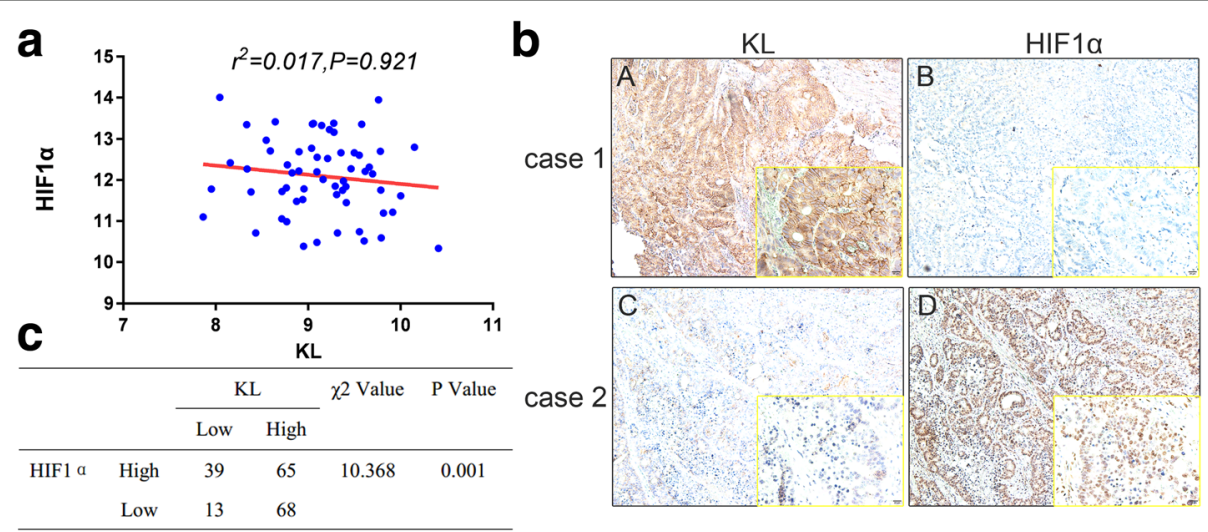

Fig. $4 \mathrm{KL}$ negatively correlated with HIF1a in CRC patients. To support the correlation obtained from in vitro assays, we first examined the mRNA of KL and HIF1a, and observed no significant correlation between $\mathrm{KL}$ and HIF1a (a). Then, we performed IHC staining in CRC patients, and observed a negative correlation between KL and HIF1a in protein level (b). Moreover, the correlation was statistically significant, indicating that KL was associated with HIF1a in protein level (c) 

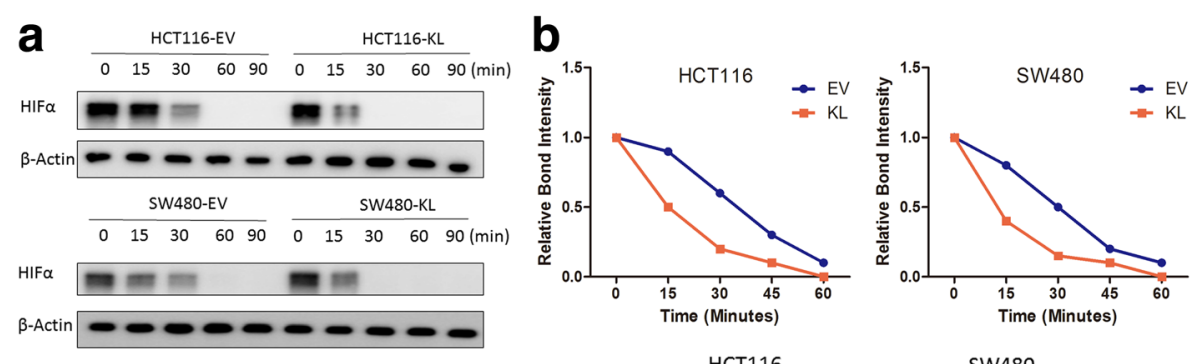

C
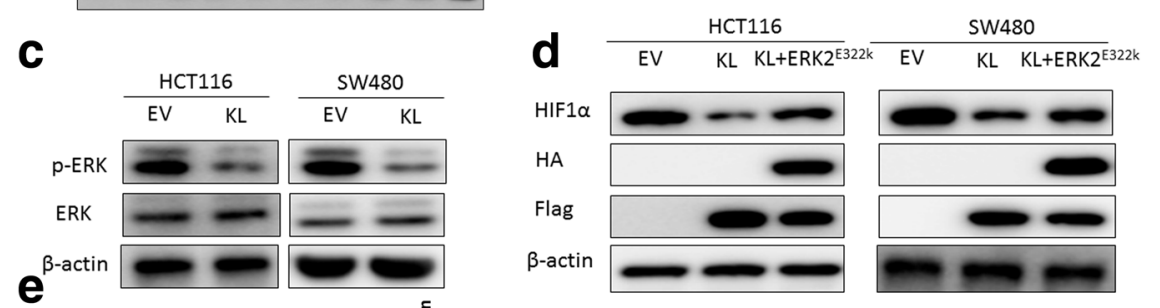

d $\mathrm{EV} \quad \mathrm{KL} \quad \mathrm{KL}+\mathrm{ERK} 2^{\mathrm{E} 322 \mathrm{k}} \mathrm{EV} \quad \mathrm{KL} \quad \mathrm{KL}+\mathrm{ERK} 2^{\mathrm{E} 322 \mathrm{k}}$
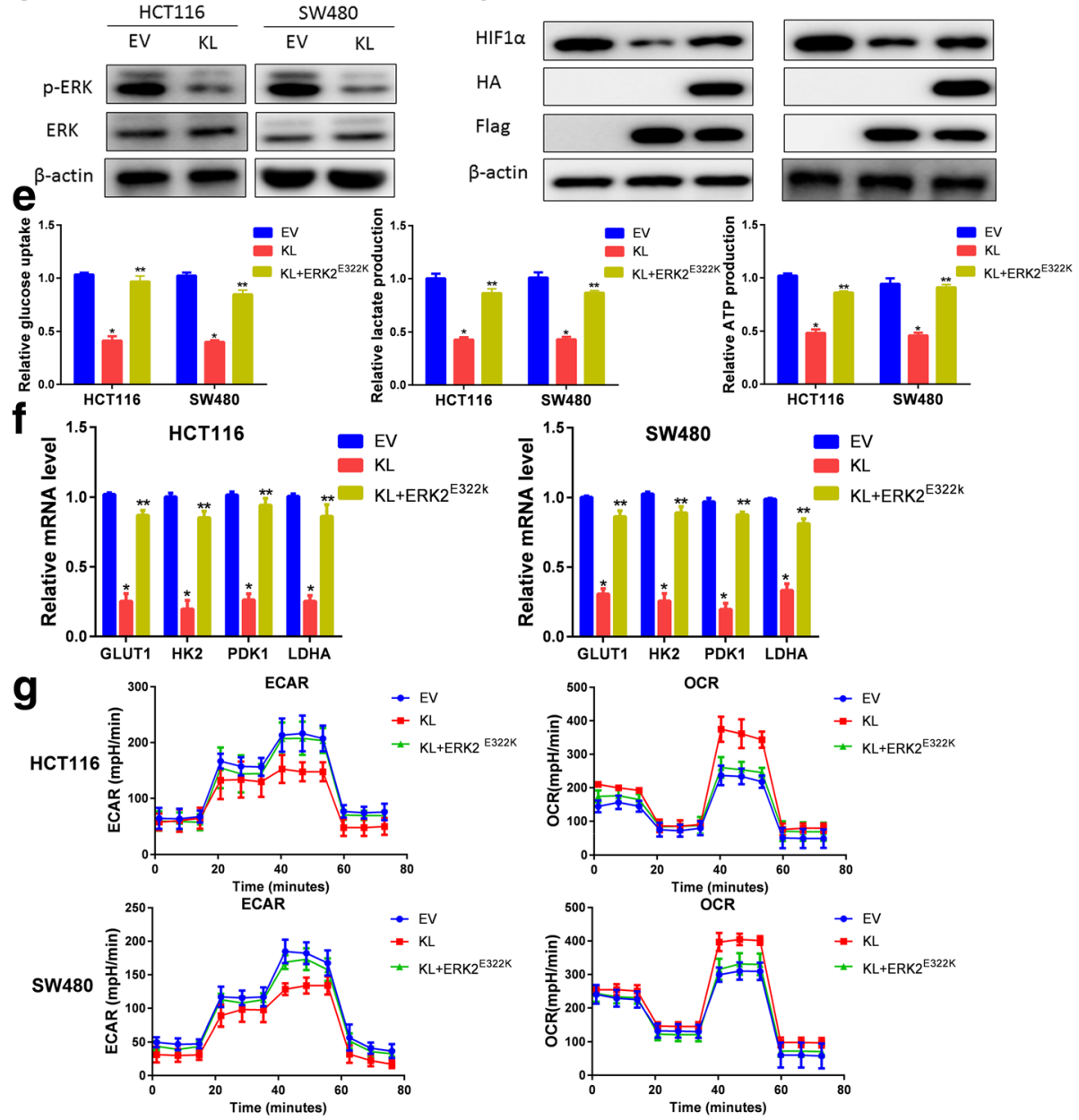

Fig. $5 \mathrm{KL}$ regulates HIF1a in an ERK dependent manner. Based on the observation that KL correlated with HIF1a in protein level instead of mRNA level, we assumed that KL might regulate HIF1a stability. First, we examined the hall-life of HIF1a in KL overexpressed HCT-116 and SW480 cells, and results indicated that KL significantly decreased the half-life of HIF1a (a and $\mathbf{b})$. To seek the underlying molecular mechanism, we examined the activation status of ERK1/2, which regulated HIF1a protein level. In KL overexpressed colon cancer cells, the activation of ERK1/2 was inhibited, indicating that KL might regulate HIF1a via ERK signaling pathway (c). To answer whether KL regulated HIF1a via ERK signaling pathway, we overexpressed constitutive activation mutant of ERK2 (ERK2 ${ }^{E 322 K}$ ) in KL overexpressed cells, and results demonstrated that ERK2 ${ }^{E 322 K}$ introduction could alleviate the decrease in HIF1a caused by KL (d). Subsequently, we examined the effect of ERK2 ${ }^{\mathrm{E} 322 \mathrm{~K}}$ on glycolysis in $\mathrm{KL}$ overexpressed cells and found ERK2 ${ }^{\mathrm{E} 322 \mathrm{~K}}$ transfection could eliminate the inhibit effect of KL on glycolysis (e). Real-time PCR analysis of HIF1a targeted glycolysis genes supported this hypothesis, as ERK2 ${ }^{E 322 K}$ introduction could partially up-regulate the mRNA level of glycolysis genes in $\mathrm{KL}$ overexpressed colon cancer cells (f). Then, we performed glycolysis analysis, and found that ERK2 $^{\text {E322K }}$ introduction could induce an increase in ECAR in KL-overexpressed colon cancer cells. In the end, the OCR analysis demonstrated that ERK2 ${ }^{\mathrm{E} 322 \mathrm{~K}}$ introduction could alleviated the attenuation in OCR caused by $\mathrm{KL}$ introduction $(\mathbf{g})$. Taken together, these results indicated that $\mathrm{KL}$ regulated aerobic glycolysis via ERK1/2 activation. ${ }^{*} P<0.05$, ** $P>0.05$ 
activity (Fig. 5f). In the end, we measured the ECAR in $\mathrm{ERK} 2^{\mathrm{E} 322 \mathrm{~K}} / \mathrm{KL}$ overexpression colon cancer cells, and demonstrated that ERK2 ${ }^{\mathrm{E} 322 \mathrm{~K}}$ overexpression increased the ECAR value in KL overexpressed cells $(P<0.05)$, suggesting the role of ERK on KL mediated glycolysis regulation (Fig. 5g). The mitochondrial respiration measurement by OCR test further supported this hypothesis, as ERK2 ${ }^{\mathrm{E} 322 \mathrm{~K}}$ introduction decreased the OCR value that caused by KL overexpression $(\mathrm{P}<0.05)$ (Fig. 5g). Taken together of the present study, we uncovered a novel function of $\mathrm{KL}$ in CRC aerobic glycolysis control, and demonstrated that ERK/ HIF1 $\alpha$ axis is responsible for KL in aerobic glycolysis regulation. These results provided novel physiological roles for KL and new aspects for the understanding of the biology and treatment of colorectal cancer.

\section{Discussion}

The KL gene was originally identified as a putative aging related suppressor gene in mice, and recovery of KL expression can be used as novel therapeutic strategies for many age-related diseases. KL is expressed most abundantly in the kidneys, but its tissue distribution also includes placenta, prostate, lung, and digestive system [36, 37]. Recent studies demonstrated that due to promoter hypermethylation, the expression level of KL was lower in tumor tissues than adjacent normal samples, indicating that KL may function as a tumor suppressor [7, 38, 39]. In consistence with these observations, the physiological role of $\mathrm{KL}$ has been widely studied, and demonstrated that KL played negative roles in oncogenesis, progression and metastasis of many cancers [40-42]. Our previous study also demonstrated that $\mathrm{KL}$ functioned as a tumor suppressor in colorectal cancer by inhibiting the IGF1R-mediated PI3K/AKT pathway [17]. Recent years have witnessed the booming of metabolism reprogramming in cancer cells, thus we questioned whether KL could also participate in metabolism control. Besides, KL was reported to regulate ageing relating processes, which also played important roles in metabolism. This also inspired us to question the contribution of KL to metabolism regulation [43-45]. What is more, the IGF1R-mediated PI3K/AKT pathway is also an important cascade in regulating metabolism [46-50]. Based on these hints, we speculated that KL might be associated with metabolism control. Thus, we performed a series of in vitro assays and examination of the correlation between KL with SUVmax reflected by PET/CT scanning, which supported our hypothesis. To search for the underlying molecular mechanism, we turned to examine its effect on HIF1 $\alpha$, a transcription factor that frequently up-regulated in solid tumors.

Solid tumor cells reside in a microenvironment far away from the blood vessels, leading to restricted nutrients and oxygen supply, thus tumor cells evolved an adaptation to survive under hypoxic conditions, known as hypoxic adaptations [51]. The best characterized metabolism reprogramming in cancer is aerobic glycolysis, which is a result of hypoxic adaptations that provided cancer cells with metabolic advantage for proliferation and metastasis. HIF1 $\alpha$ played central roles in hypoxic adaptation, and received much attention since its discovery [52]. HIF1 $\alpha$ is strictly regulated by a series of post-translational modifications, including acetylation, hydroxylation, phosphorylation and ubiquitination [32, 53]. Previous studies demonstrated that hypoxia activation could induce activation of ERK signaling pathway, and moreover, ERK activity regulated the protein stability of HIF1 $\alpha$ [54-56]. Thus, we examined the activation status of ERK1/2 in KL overexpressed colon cancer cells, and observed a decrease in the activation of ERK1/2. Subsequent assays demonstrated that KL regulated HIF1 $\alpha$ and glycolysis in an ERK dependent manner. However, there are some issues needed to be addressed in the future, for example, whether the expression of KL changed upon hypoxia, and whether hypoxia lead to epigenetic changes in the promoter region of KL. Furthermore, the impact of ERK activation on $\mathrm{KL}$ expression was not examined in the present study, and this also needs to be addressed in the future.

\section{Conclusions}

Taken together, our present study uncovered a novel function of tumor suppressor, $\mathrm{KL}$, in glycolysis regulation and provided the possible molecular mechanism. These results identified KL as a novel target for the diagnosis and treatment for colorectal cancer. Moreover, attempts to target cancer cell metabolism are also novel strategies for the treatment of colorectal cancer, and cutting fuel supply may provide a thoroughly novel aspect in targeting colorectal cancer.

\section{Additional files}

Additional file 1: Table S1. Baseline clinicopathological features for patients in PET/CT and RNA study. Table S2. Primer sequences used in the study. (DOCX $20 \mathrm{~kb}$ )

Additional file 2: Figure S1. Representative pictures of each score for immunohistochemical staining results of KL in TMA of FUSCC. (TIF $2829 \mathrm{~kb}$ )

Additional file 3: The clinicopathological information and

immunohistochemistry results in the TMA cohort of 185 patients. (XLSX $35 \mathrm{~kb})$

\section{Abbreviations}

CRC: Colorectal cancer; ECAR: extracellular acidification rate; FUSCC: Fudan University Shanghai Cancer Center; HIF1: Hypoxia-inducible factor 1; IHC: Immunohistochemical Staining; IRS: immunoreactivity score; KL: Klotho; OCR: Oxygen consumption rate; SUVmax: maximum standardized uptake value

\section{Funding}

This research was supported by the National Science Foundation of China (No. 81772599, 81702353) and Shanghai Municipal Natural Science Foundation (17ZR1406400). The funders had no role in the study design, data collection and analysis, decision to publish, or preparation of the manuscript. 


\section{Availability of data and materials}

All data generated or analyzed during this study are included in this published article. Additional material presented in the current study is available from the corresponding authors on reasonable request.

\section{Authors' contributions}

QGL and XXL conceived this study. YQL, QGL and LL improved the study design and contributed to the interpretation of results. JL, DKL, QL, and SJC performed the study. QGL and YQL performed data processing and statistical analysis. QGL and LL wrote the manuscript. YLL revised the manuscript and approved the final version. All authors read and approved the final manuscript.

\section{Ethics approval and consent to participate}

Clinical colorectal cancer samples were obtained from the Fudan University Shanghai Cancer Center, with consents from the patients and the study protocol was approved by the Ethics Committee of the Fudan University Shanghai Cancer Center, Shanghai, China.

\section{Competing interests}

The authors declare that they have no competing interests.

\section{Publisher's Note}

Springer Nature remains neutral with regard to jurisdictional claims in published maps and institutional affiliations.

\section{Author details}

'Department of Colorectal Surgery, Fudan University Shanghai Cancer Center, No.270 Dong'an Road, Xuhui District, Shanghai 200032, China. ${ }^{2}$ Department of Oncology, Shanghai Medical College, Fudan University, No.270 Dong'an Road, Xuhui District, Shanghai 200032, China. ${ }^{3}$ Departments of CyberKnife, Huashan Hospital, Fudan University, Shanghai 200032, China.

\section{Received: 19 March 2018 Accepted: 28 May 2018}

Published online: 08 June 2018

\section{References}

1. Torre LA, Bray F, Siegel RL, Ferlay J, Lortet-Tieulent J, Jemal A. Global cancer statistics, 2012. CA Cancer J Clin. 2015;65:87-108

2. Kouzminova N, Lu T, Lin AY. Molecular basis of colorectal cancer. N Engl J Med. 2010:362:1245-6. author reply 1246-1247

3. Sugai T, Habano W. Pathological diagnosis and its molecular basis in colorectal Cancer. Gan To Kagaku Ryoho. 2016;43:294-9.

4. Kim HR, Nam BY, Kim DW, Kang MW, Han JH, Lee MJ, Shin DH, Doh FM, Koo HM, Ko Kl, et al. Circulating alpha-klotho levels in CKD and relationship to progression. American journal of kidney diseases : the official journal of the National Kidney Foundation. 2013;61:899-909.

5. Goetz R, Beenken A, Ibrahimi OA, Kalinina J, Olsen SK, Eliseenkova AV, Xu C, Neubert TA, Zhang F, Linhardt RJ, et al. Molecular insights into the klothodependent, endocrine mode of action of fibroblast growth factor 19 subfamily members. Mol Cell Biol. 2007;27:3417-28.

6. Yamamoto M, Clark JD, Pastor JV, Gurnani P, Nandi A, Kurosu H, Miyoshi M, Ogawa Y, Castrillon DH, Rosenblatt KP, Kuro-o M. Regulation of oxidative stress by the anti-aging hormone klotho. J Biol Chem. 2005;280:38029-34.

7. Pan J, Zhong J, Gan LH, Chen SJ, Jin HC, Wang X, Wang LJ. Klotho, an antisenescence related gene, is frequently inactivated through promoter hypermethylation in colorectal cancer. Tumour Biol. 2011:32:729-35.

8. Perveez M, Ajaz M, Afroze D. Promoter hypermethylation of KLOTHO; an anti-senescence related gene in colorectal cancer patients of Kashmir valley. Mol Biol Res Commun. 2015;4:217-24.

9. Azuma M, Koyama D, Kikuchi J, Yoshizawa H, Thasinas D, Shiizaki K, Kuro-o M, Furukawa Y, Kusano E. Promoter methylation confers kidney-specific expression of the klotho gene. FASEB J. 2012;26:4264-74.

10. Usuda J, Ichinose S, Ishizumi T, Ohtani K, Inoue T, Saji H, Kakihana M, Kajiwara N, Uchida O, Nomura M, et al. Klotho predicts good clinical outcome in patients with limited-disease small cell lung cancer who received surgery. Lung Cancer. 2011;74:332-7.

11. Tang X, Wang Y, Fan Z, Ji G, Wang M, Lin J, Huang S, Meltzer SJ. Klotho: a tumor suppressor and modulator of the Wnt/beta-catenin pathway in human hepatocellular carcinoma. Lab Investig. 2016;96: 197-205.
12. Xie B, Zhou J, Yuan L, Ren F, Liu DC, Li Q, Shu G. Epigenetic silencing of klotho expression correlates with poor prognosis of human hepatocellular carcinoma. Hum Pathol. 2013;44:795-801.

13. Lu L, Katsaros D, Wiley A, de la Longrais IA, Puopolo M, Yu H. Klotho expression in epithelial ovarian cancer and its association with insulin-like growth factors and disease progression. Cancer Investig. 2008;26:185-92.

14. Zhou X, Wang X. Klotho: a novel biomarker for cancer. J Cancer Res Clin Oncol. 2015;141:961-9.

15. Wolf I, Levanon-Cohen S, Bose S, Ligumsky H, Sredni B, Kanety H, Kuro-o M, Karlan B, Kaufman B, Koeffler HP, Rubinek T. Klotho: a tumor suppressor and a modulator of the IGF-1 and FGF pathways in human breast cancer. Oncogene. 2008;27:7094-105.

16. Doi S, Zou Y, Togao O, Pastor JV, John GB, Wang L, Shiizaki K, Gotschall R, Schiavi S, Yorioka N, et al. Klotho inhibits transforming growth factor-beta1 (TGF-beta1) signaling and suppresses renal fibrosis and cancer metastasis in mice. J Biol Chem. 2011;286:8655-65.

17. Li XX, Huang LY, Peng JJ, Liang L, Shi DB, Zheng HT, Cai SJ. Klotho suppresses growth and invasion of colon cancer cells through inhibition of IGF1R-mediated PI3K/AKT pathway. Int J Oncol. 2014;45:611-8.

18. Mattoo RL. The roles of fibroblast growth factor (FGF)-23, alpha-klotho and Furin protease in calcium and phosphate homeostasis : a mini-review. Indian J Clin Biochem. 2014;29:8-12.

19. Urakawa I, Yamazaki Y, Shimada T, lijima K, Hasegawa H, Okawa K, Fujita T, Fukumoto S, Yamashita T. Klotho converts canonical FGF receptor into a specific receptor for FGF23. Nature. 2006;444:770-4.

20. Taylor MA, Lee YH, Schiemann WP. Role of TGF-beta and the tumor microenvironment during mammary tumorigenesis. Gene Expr. 2011;15: $117-32$.

21. Joshi A, Cao D. TGF-beta signaling, tumor microenvironment and tumor progression: the butterfly effect. Front Biosci (Landmark Ed). 2010;15: 180-94.

22. Huang D, Du X. Crosstalk between tumor cells and microenvironment via Wnt pathway in colorectal cancer dissemination. World I Gastroenterol. 2008;14:1823-7.

23. Sanchez-Lopez E, Flashner-Abramson E, Shalapour S, Zhong Z, Taniguchi K, Levitzki A, Karin M. Targeting colorectal cancer via its microenvironment by inhibiting IGF-1 receptor-insulin receptor substrate and STAT3 signaling. Oncogene. 2016;35:2634-44.

24. Katoh M. FGFR inhibitors: effects on cancer cells, tumor microenvironment and whole-body homeostasis (review). Int J Mol Med. 2016;38:3-15.

25. Warburg O. On the origin of cancer cells. Science. 1956;123:309-14.

26. Cairns RA, Harris IS, Mak TW. Regulation of cancer cell metabolism. Nat Rev Cancer. 2011;11:85-95.

27. Wallace DC. Mitochondria and cancer. Nat Rev Cancer. 2012;12:685-98.

28. Keith B, Johnson RS, Simon MC. HIF1alpha and HIF2alpha: sibling rivalry in hypoxic tumour growth and progression. Nat Rev Cancer. 2011;12:9-22.

29. Weidemann A, Johnson RS. Biology of HIF-1alpha. Cell Death Differ. 2008;15: $621-7$.

30. Yee Koh M, Spivak-Kroizman TR, Powis G. HIF-1 regulation: not so easy come, easy go. Trends Biochem Sci. 2008;33:526-34.

31. Brahimi-Horn MC, Chiche J, Pouyssegur J. Hypoxia signalling controls metabolic demand. Curr Opin Cell Biol. 2007;19:223-9.

32. Bertout JA, Patel SA, Simon MC. The impact of $\mathrm{O} 2$ availability on human cancer. Nat Rev Cancer. 2008;8:967-75.

33. Li Q, Wu J, Wei P, Xu Y, Zhuo C, Wang Y, Li D, Cai S. Overexpression of forkhead box C2 promotes tumor metastasis and indicates poor prognosis in colon cancer via regulating epithelial-mesenchymal transition. Am J Cancer Res. 2015;5:2022-34.

34. Li Y, Liang L, Dai W, Cai G, Xu Y, Li X, Li Q, Cai S. Prognostic impact of programed cell death-1 (PD-1) and PD-ligand 1 (PD-L1) expression in cancer cells and tumor infiltrating lymphocytes in colorectal cancer. Mol Cancer. 2016;15:55.

35. Emerling BM, Weinberg F, Liu JL, Mak TW, Chandel NS. PTEN regulates p300-dependent hypoxia-inducible factor 1 transcriptional activity through Forkhead transcription factor 3a (FOXO3a). Proc Natl Acad Sci U S A. 2008; 105:2622-7.

36. Kuro-o M. Klotho in health and disease. Curr Opin Nephrol Hypertens. 2012; 21:362-8

37. Martin-Nunez E, Donate-Correa J, Muros-de-Fuentes M, Mora-Fernandez C, Navarro-Gonzalez JF. Implications of klotho in vascular health and disease. World J Cardiol. 2014;6:1262-9. 
38. Dallol A, Buhmeida A, Merdad A, Al-Maghrabi J, Gari MA, Abu-Elmagd MM, Elaimi A, Assidi M, Chaudhary AG, Abuzenadah AM, et al. Frequent methylation of the KLOTHO gene and overexpression of the FGFR4 receptor in invasive ductal carcinoma of the breast. Tumour Biol. 2015;36: 9677-83.

39. Wang L, Wang $X$, Wang $X$, Jie $P$, Lu H, Zhang $S$, Lin X, Lam EK, Cui Y, Yu J, $\mathrm{Jin} \mathrm{H}$. Klotho is silenced through promoter hypermethylation in gastric cancer. Am J Cancer Res. 2011;1:111-9.

40. Lojkin I, Rubinek T, Orsulic S, Schwarzmann O, Karlan BY, Bose S, Wolf I. Reduced expression and growth inhibitory activity of the aging suppressor klotho in epithelial ovarian cancer. Cancer Lett. 2015;362:149-57.

41. Xie B, Zhou J, Shu G, Liu DC, Zhou J, Chen J, Yuan L. Restoration of klotho gene expression induces apoptosis and autophagy in gastric cancer cells: tumor suppressive role of klotho in gastric cancer. Cancer Cell Int. 2013;13:18.

42. Chen B, Ma X, Liu S, Zhao W, Wu J. Inhibition of lung cancer cells growth, motility and induction of apoptosis by klotho, a novel secreted Wnt antagonist, in a dose-dependent manner. Cancer Biol Ther. 2012;13:1221-8.

43. Chen J, Lin Y, Sun Z. Deficiency in the anti-aging gene klotho promotes aortic valve fibrosis through AMPKalpha-mediated activation of RUNX2. Aging Cell. 2016;15:853-60

44. Xu Y, Sun Z. Molecular basis of klotho: from gene to function in aging. Endocr Rev. 2015;36:174-93.

45. Sopjani M, Rinnerthaler M, Kruja J, Dermaku-Sopjani M. Intracellular signaling of the aging suppressor protein klotho. Curr Mol Med. 2015;15:27-37.

46. Sadagurski M, White MF. Integrating metabolism and longevity through insulin and IGF1 signaling. Endocrinol Metab Clin N Am. 2013;42:127-48.

47. Li X, Monks B, Ge Q, Birnbaum MJ. Akt/PKB regulates hepatic metabolism by directly inhibiting PGC-1alpha transcription coactivator. Nature. 2007;447: 1012-6.

48. Osorio J. Metabolism: an Akt-independent pathway for regulation of gluconeogenesis. Nat Rev Endocrinol. 2012;8:257.

49. Lu M, Wan M, Leavens KF, Chu Q, Monks BR, Fernandez S, Ahima RS, Ueki K, Kahn CR, Birnbaum MJ. Insulin regulates liver metabolism in vivo in the absence of hepatic Akt and Foxo1. Nat Med. 2012;18:388-95.

50. Long YC, Cheng Z, Copps KD, White MF. Insulin receptor substrates Irs1 and Irs2 coordinate skeletal muscle growth and metabolism via the Akt and AMPK pathways. Mol Cell Biol. 2011:31:430-41.

51. Rankin EB, Giaccia AJ. The role of hypoxia-inducible factors in tumorigenesis. Cell Death Differ. 2008;15:678-85.

52. Koppenol WH, Bounds PL, Dang CV. Otto Warburg's contributions to current concepts of cancer metabolism. Nat Rev Cancer. 2011;11:325-37.

53. Kaelin WG. Proline hydroxylation and gene expression. Annu Rev Biochem. 2005;74:115-28.

54. Minet E, Arnould T, Michel G, Roland I, Mottet D, Raes M, Remacle J, Michiels C. ERK activation upon hypoxia: involvement in HIF-1 activation. FEBS Lett. 2000;468:53-8,

55. Lim JH, Lee ES, You HJ, Lee JW, Park JW, Chun YS. Ras-dependent induction of HIF-1alpha785 via the Raf/MEK/ERK pathway: a novel mechanism of Rasmediated tumor promotion. Oncogene. 2004;23:9427-31.

56. Mylonis I, Chachami G, Samiotaki M, Panayotou G, Paraskeva E, Kalousi A, Georgatsou E, Bonanou S, Simos G. Identification of MAPK phosphorylation sites and their role in the localization and activity of hypoxia-inducible factor-1alpha. J Biol Chem. 2006;281:33095-106.

\section{Ready to submit your research? Choose BMC and benefit from:}

- fast, convenient online submission

- thorough peer review by experienced researchers in your field

- rapid publication on acceptance

- support for research data, including large and complex data types

- gold Open Access which fosters wider collaboration and increased citations

- maximum visibility for your research: over $100 \mathrm{M}$ website views per year

At BMC, research is always in progress.

Learn more biomedcentral.com/submissions 